\title{
Vortex Panel Method in Axisymmetric Cylindrical Coordinate
}

\author{
Masaya Kato $^{1}$, Suguru Shiratori ${ }^{1}$, Hideaki Nagano ${ }^{1}$, Kenjiro Shimano ${ }^{1}$ \\ ${ }^{1}$ Dept., Mechanical Systems Engineering, Tokyo City University \\ 1-28-1 Tamazutsumi, Setagaya-ku, Tokyo 158-8557 Japan \\ g2081014@tcu.ac.jp (M. Kato); sshrator@tcu.ac.jp (S. Shiratori)
}

\section{Extended Abstract}

A ducted fan is used in various product such as a radio-controlled airplane and a ventilation system. Recently, it has been considered as an option for a hyperloop thruster. Generally, the thrust of the ducted fan decreases when the vehicle is moving. By modifying the shape of the duct, the characteristics of thrust decrease can be changed. For narrow duct, the lager thrust can be achieved even for higher translational velocity of the vehicle. Therefore, there is a demand to optimize the duct shape according to the application situation. In the optimization procedure, calculation of the aerodynamic performance is necessary for a given shape. When we use general purpose CFD analysis, it takes large computational cost to obtain the aerodynamic performance. Therefore, an alternative efficient calculation procedure is required.

As a lightweight calculation procedure, this study focuses on the vortex panel method in axisymmetric cylindrical coordinates, because ducted fans are nearly axisymmetric. The panel method is a kind of boundary element method and is a solution procedure for an inviscid and incompressible flows. It is a lightweight calculation because it represents the actual flow by combining the vortex distribution on the object surface and the uniform flow. The unknowns are only needed on the boundary without any spatial mesh on the bulk domain. In the Cartesian coordinate system, a number of research on the panel method has been carried out for a long time, but there is no report implementing the axisymmetric panel method yet.

To construct the axisymmetric panel method, it is necessary to derive the Green's function and to establish a method to consider the effect of viscous flow. In this study, we derived the Green's function for the governing equation of panel method in axisymmetric cylindrical coordinate system by modification of the Green's function for the three-dimensional cylindrical coordinate obtained by Cohl \& Tholine [1]. The Green's function obtained in the present study is very compact, which is composed by the single wave number, whereas in other problems, the Green's function for the cylindrical coordinate often composed by the Bessel series expansion, which requires the infinite sum of series. In this sense, the lightweight panel method is realized in the present study.

Regarding the effect of viscous flow, we solve the boundary layer equations coupled with the inviscid panel method, according to the conventional panel method for Cartesian coordinate [2]. First, we calculate the inviscid flow by the panel method, then we solve the boundary layer equations by letting the outer-layer velocity profile be the solution of the inviscid flow. Next, the location of the object is displaced with adding the boundary layer thickness, then the inviscid flow is recalculated. These procedures are repeated until the solution converges.

The validity of the proposed method was confirmed through the two problems; the flow past a sphere, whose solution can be analytically obtained, and the flow past the duct shape, whose solution is numerically obtained by the finite volume method. For both cases, the solution obtained by the present panel method showed sufficient agreement with the solutions by other methods. By the proposed panel method, lightweight calculation of the shape optimization can be realized not only for ducted fans but also for general axisymmetric objects.

\section{References}

[1] Howard S. Cohl, Joel E. Tholine, “A compact cylindrical green's function expansion for the solution of potential problems" The Astrophysical Journal 527:86-101, 1999.

[2] Mark Drela, "Viscous-inviscid analysis of transonic and low Reynolds number airfoils" AIAA Journal Vol. 25, No. 10, 1987. 